\title{
Pinning and gas oversaturation imply stable single surface nanobubbles
}

\author{
Detlef Lohse ${ }^{1, *}$ and Xuehua Zhang (张雪花 $)^{2,1}$ \\ ${ }^{1}$ Physics of Fluids group, Department of Science and Technology, Mesa+ Institute, and J. M. Burgers Centre for Fluid Dynamics, \\ University of Twente, P.O. Box 217, 7500 AE Enschede, The Netherlands \\ ${ }^{2}$ School of Civil, Environmental and Chemical Engineering, RMIT University, Melbourne, VIC 3001, Australia
}

(Received 4 November 2014; published 27 March 2015)

\begin{abstract}
Surface nanobubbles are experimentally known to survive for days at hydrophobic surfaces immersed in gas-oversaturated water. This is different from bulk nanobubbles, which are pressed out by the Laplace pressure against any gas oversaturation and dissolve in submilliseconds, as derived by Epstein and Plesset [J. Chem. Phys. 18, 1505 (1950)]. Pinning of the contact line has been speculated to be the reason for the stability of the surface nanobubbles. Building on an exact result by Popov [Phys. Rev. E 71, 036313 (2005)] on coffee stain evaporation, here we confirm this speculation by an exact calculation for single surface nanobubbles. It is based only on (i) the diffusion equation, (ii) Laplace pressure, and (iii) Henry's equation, i.e., fluid dynamical equations which are all known to be valid down to the nanometer scale. The crucial parameter is the gas oversaturation $\zeta$ of the liquid. At the stable equilibrium, the gas overpressures due to this oversaturation and the Laplace pressure balance. The theory predicts how the contact angle of the pinned bubble depends on $\zeta$ and the surface nanobubble's footprint lateral extension $L$. It also predicts an upper lateral extension threshold for stable surface nanobubbles to exist.
\end{abstract}

DOI: 10.1103/PhysRevE.91.031003

PACS number(s): 47.55.D-, 66.10.C-

Surface nanobubbles (for recent reviews see [1-5]) are long-living nanoscale spherical cap-shaped bubbles on a solid surface in contact with gas oversaturated water. Their existence and gaseous nature has meanwhile been confirmed with various methods. It has however been a challenge to understand their very long lifetime - up to days, rather than the microseconds one expects given their small size and the resulting large Laplace pressure inside the nanobubble, which presses out the nanobubbles against any oversaturation. This expectation originates from and is substantiated by the exact seminal result by Epstein and Plesset [6], who derived the full diffusional dynamics and thus in particular also the lifetime of spherical bulk bubbles of any size for any imposed external gas concentration from (i) the diffusion equation, (ii) Laplace pressure, and (iii) Henry's law. They find that the lifetime of a single spherical bulk bubble of initial radius $R_{0}$ in a liquid with gas concentration $c_{\infty}$ far away from the bubble and gas solubility $c_{s}$ is ${ }^{1}$

$$
\tau_{\text {life }} \approx \begin{cases}\frac{R_{0}^{2} \rho_{g}}{2 D c_{s}|\zeta|} & \text { for large } R_{0} \text { and } \zeta<0 \\ \frac{R_{0}^{2} \rho_{g}}{3 D c_{s}} & \text { for small } R_{0} \text { and any } \zeta\end{cases}
$$

Here $D$ is the diffusion constant, $\rho_{g}$ is the gas density, and

$$
\zeta=\frac{c_{\infty}}{c_{s}}-1
$$

is the gas oversaturation. "Large" refers to bubbles in which the internal pressure (neglecting vapor pressure)

$$
p_{g}(t)=P_{0}+\frac{2 \sigma}{R(t)}
$$

\footnotetext{
*d.lohse@utwente.nl

${ }^{1}$ Epstein and Plesset in fact also calculate the nearly indistinguishable exact result, which includes short transients, by numerically solving an ODE, but for the discussion here the given approximate result is sufficient.
}

is dominated by the ambient pressure $P_{0}$, i.e., $R \gg 2 \sigma / P_{0}$, where $\sigma$ is the surface tension, and "small" refers to bubbles in which the Laplace pressure is dominant $\left(R \ll 2 \sigma / P_{0} \approx\right.$ $1.4 \mu \mathrm{m}$ for air bubbles in water under ambient pressure) and at the same time $R \ll 2 \sigma /\left[P_{0}(\zeta+1)\right]$. For gas oversaturation $\zeta>0$ large bubbles even grow (which Epstein and Plesset also exactly calculated). In contrast, small bubbles are pressed out by the Laplace pressure and shrink for any oversaturation $\zeta>0$. This is why no stable nanobubbles can exist in the bulk. $^{2}$

So how come that surface nanobubbles are then stable for many days? Recent findings have suggested that the pinning of the three-phase nanobubble-liquid-surface contact line is very crucial for their long lifetimes [7-10]. The experimental evidence for contact line pinning of surface nanobubbles is very strong, see, e.g., Refs. [11,12]. The pinning results from chemical and geometrical surface heterogeneities, see e.g. the reviews [13-17]. Such heterogeneities are omnipresent and unavoidable on any solid surface. They also lead to contact angle hysteresis, i.e., the difference between an advancing and a receding contact angle $[18,19]$.

References [7-10] have realized both the important role of pinning for the stabilization of the nanobubbles and also that their lifetime is determined by the diffusion process with the outer world. Liu and Zhang [9,10] even offer kinetic lattice density functional calculations and MD simulations to demonstrate this.

In this present paper we actually analytically calculate the equilibrium contact angle and demonstrate that only gas oversaturation can lead to stable single surface bubbles, provided that they are small enough. We will present an exact calculation, showing how exactly pinning stabilizes a single

\footnotetext{
${ }^{2}$ Note that for very strong oversaturation $\zeta \gg 1$ this small bubble limit only applies to very small bubbles with $R \ll 2 \sigma /\left[P_{0}(\zeta+1)\right] \ll$ $2 \sigma / P_{0}$. So by strongly gas-oversaturating liquids one can stabilize small bulk bubbles with $R>2 \sigma /\left[P_{0}(\zeta+1)\right]$.
} 

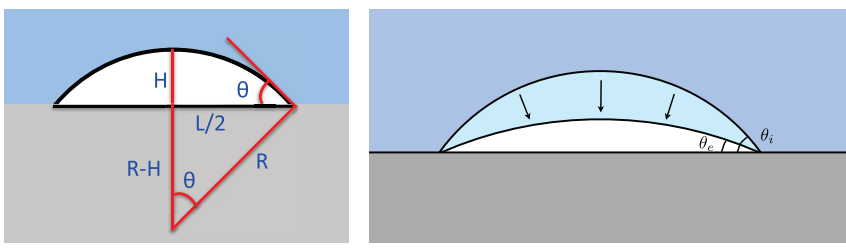

FIG. 1. (Color online) (a) Sketch of a spherical cap-shaped surface nanobubbles and definition of the parameters describing the surface nanobubble's geometry, namely the nanobubble's footprint lateral extension $L$ (or its footprint radius $L / 2$ ), its height $H$, its contact angle $\theta$ on the gas side, and its radius of curvature $R=\left(L^{2}+4 H^{2}\right) /(8 H)=L /(2 \sin \theta)$. (b) Sketch of the shrinking process of a pinned surface nanobubbles with initial contact angle $\theta_{i}$ : Due to the pinning, $L$ is fixed. Shrinking thus implies a decrease in $\theta$ and $H$ and an increase in the radius of curvature. Therefore, the Laplace pressure inside the bubble reduces and eventually becomes too weak to further press out the bubble against the oversaturation. The contact angle $\theta_{e}<\theta_{i}$ of this stable equilibrium state depends on $\zeta$ and $L$. Surface nanobubbles with initially $\theta_{i}<\theta_{e}$ will grow towards $\theta_{e}$.

surface nanobubble in an oversaturated liquid and how the resulting equilibrium contact angle $\theta_{e}$ of the stable surface nanobubble depends on its lateral extension $L$ and on the gas oversaturation $\zeta>0$. The calculation is based only on (i) the diffusion equation, (ii) Laplace pressure, and (iii) Henry's equation, i.e., fluid dynamical equations which are all known to be valid down to the nanometer scale, see, e.g., the review [20]. Advection effects, which will emerge for strong stirring of the liquid, are neglected.

A sketch of the spherical cap-shaped surface bubble, including our notation, is shown in Fig. 1. The bubble volume $V=\pi H\left(3 L^{2}+4 H^{2}\right) / 24$ follows from the bubble's lateral diameter $L$ (which is fixed in time, assuming perfect pinning) and its height $H$. The contact angle $\theta$ is given by $\sin \theta=$ $4 L H /\left(L^{2}+4 H^{2}\right)$. The bubble's radius of curvature, which determines its Laplace pressure, ${ }^{3}$ is

$$
R=L /(2 \sin \theta) .
$$

For an air bubble dissolution (or growth) the air diffusion in the liquid is the rate-limiting process. ${ }^{4}$ We can therefore consider the quasistatic limit, in which the gas concentration field $c(r, z)$ around the bubble is described by the quasistatic diffusion equation

$$
\partial_{t} c=D \nabla^{2} c \approx 0
$$

\footnotetext{
${ }^{3}$ While Zhang et al. [7] provided very strong and convincing experimental evidence for pinning, in the theoretical part of their paper they erroneously assumed that the gas pressure inside the bubble would be constant, ignoring the time-dependent Laplace contribution to the pressure. This wrong assumption implies that Henry's law Eq. (6) simply gives $c_{s}$ for the gas concentration just outside the bubble, i.e., the feedback mechanism is absent. This absence implies an unstable equilibrium for the surface nanobubble (see Eq. (12) of Ref. [7]), in contrast to the stable equilibrium resulting from the correct calculation of this present paper.

${ }^{4}$ In the case of the extremely soluble $\mathrm{CO}_{2}$ corrections may arise [28].
}

where $r$ and $z$ are the radial and vertical coordinates, respectively, and where axial symmetry has been assumed. At the bubble-water interface, the gas concentration is given by Henry's law,

$$
c(R, t)=\frac{c_{s}}{P_{o}} p_{g}(t)=\frac{c_{s}}{P_{o}}\left(P_{0}+\frac{4 \sigma \sin \theta}{L}\right) .
$$

Far away from the bubble $c(z \rightarrow \infty)=c_{\infty}$, whereas the noflux condition $\partial c / \partial z=0$ applies on the substrate surface $z=$ 0 . This fully defines the problem. ${ }^{5}$

The calculation leading to an exact solution of this problem is analog to Popov's [21] seminal exact solution of the "coffee stain evaporation problem" [22], which is also diffusion controlled and in which the coffee stain is also pinned. For an early calculation of the evaporation of a pinned droplet we also refer to Ref. [23]. Extending Popov's calculation [21], which perfectly describes the experimentally measured evaporation rate of a pinned coffee stain [24,25], to the present case of the diffusive shrinkage or growth of a surface bubble finally gives the change rate $d M / d t$ of the bubble mass $M$ as

$$
\frac{d M}{d t}=-\frac{\pi}{2} L D\left[\left(P_{0}+\frac{4 \sigma \sin \theta}{L}\right) \frac{c_{s}}{P_{0}}-c_{\infty}\right] f(\theta),
$$

with

$$
f(\theta)=\frac{\sin \theta}{1+\cos \theta}+4 \int_{0}^{\infty} \frac{1+\cosh 2 \theta \xi}{\sinh 2 \pi \xi} \tanh [(\pi-\theta) \xi] d \xi
$$

(which is positive definite). The bubble mass $M(t)$ itself can be expressed in terms of the gas density $\rho_{g}$, its (fixed) footprint diameter $L$, and its contact angle $\theta(t)$ as

$$
M=\rho_{g} \frac{\pi}{8} L^{3} \frac{\cos ^{3} \theta-3 \cos \theta+2}{3 \sin ^{3} \theta} .
$$

Equations (7) and (9) yield an ODE for $\theta(t)$, namely

$$
\frac{d \theta}{d t}=-\frac{4 D}{L^{2}} \frac{c_{s}}{\rho_{g}}(1+\cos \theta)^{2} f(\theta)\left[\frac{L_{c}}{L} \sin \theta-\zeta\right],
$$

with the critical lateral extension $L_{c}=4 \sigma / P_{0} \approx 2.84 \mu \mathrm{m}$ for air bubbles in water under ambient conditions. Equation (10) can straightforwardly be solved numerically.

Before doing so, we however first discuss the physical implications of this result (10). One immediately sees that for gas undersaturation $-1 \leqslant \zeta<0$ no stable surface bubble can exist, as then the right-hand side of Eq. (10) and thus the shrinkage rate $d \theta / d t$ is always negative and the surface bubble dissolves down to $\theta=0 .{ }^{6}$ However, for (fixed) gas oversaturation $\zeta>0$ a stable equilibrium (i.e., $d \theta / d t=0$ for the corresponding equilibrium contact angle $\theta_{e}$ ) can exist, namely for

$$
\sin \theta_{e}=\zeta \frac{L}{L_{c}}
$$

\footnotetext{
${ }^{5}$ Note that in Eq. (6) we have assumed the disjoining pressure to be irrelevant.

${ }^{6}$ Cases in which surface nanobubbles on first sight seem to have survived (partial) degassing of the water later turned out not to be surface nanobubbles but surface nanodroplets of some liquid with low solubility in water [33].
} 

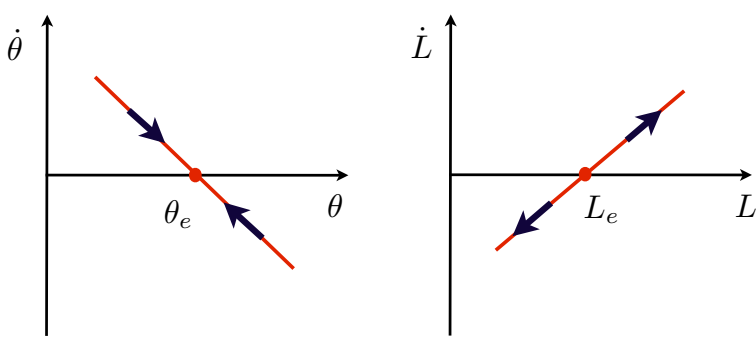

FIG. 2. (Color online) (a) Sketch of the stable equilibrium $\theta_{e}$ for pinned surface nanobubbles in the $\dot{\theta}$ vs $\theta$ phase space, cf. Eq. (10). A sketch of the bubble height phase space $\dot{H}$ vs $H$ looks the same. (b) Sketch of the unstable equilibrium $L_{e}$ for unpinned surface nanobubbles with constant contact angle in the $\dot{L}$ vs $L$ phase space, cf. Eq. (15). For bulk bubbles, the sketch looks the same, with the lateral extension $L$ then meaning the bubble diameter.

Indeed, the equilibrium is stable, as one can see from writing the big bracket in Eq. $(10)$ as $[\cdots]=\left(\sin \theta-\sin \theta_{e}\right) L_{c} / L$ : Surface bubbles with $\theta>\theta_{e}$ shrink and those with $\theta<\theta_{e}$ grow, see the sketch of the phase space in Fig. 2(a). The balance corresponding to this stable equilibrium reflects the competition between the gas influx into the surface nanobubble due to the gas oversaturation and the outflux due to the Laplace pressure.

Equation (11) [together with Eq. (10)] is the main result of this paper. It gives the functional dependence $\theta_{e}(\zeta, L)$ of the equilibrium contact angle, which is shown in Fig. 3. For fixed pinning lateral extension $L$ the equilibrium contact angle increases with the gas oversaturation $\zeta$, as one would expect. For the small contact angles relevant for surface nanobubbles this dependence is basically linear for all practical purposes. Vice versa, for fixed oversaturation $\zeta>0$ the surface nanobubbles with a larger lateral extension have a larger equilibrium contact angle, which indeed is seen in various experiments, see, e.g., [26] or Fig. 6 of [27]. From the slope of such plots $\sin \theta_{e}$ vs $L$ one can even read off the applied gas oversaturation as $\zeta=$ slope $\times L_{c}$. Note that the size dependence of the contact angle thus has nothing

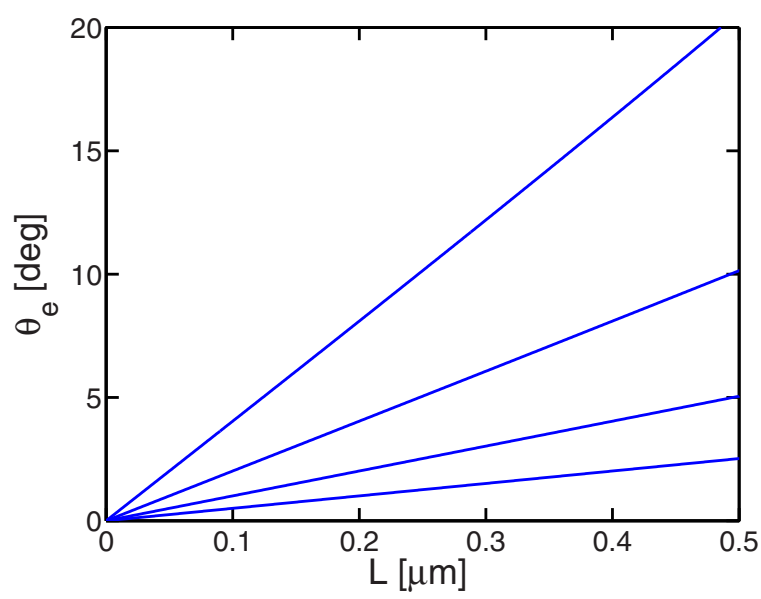

FIG. 3. (Color online) Equilibrium contact angle $\theta_{e}$ of a nitrogen bubble in water as function of the lateral footprint size $L$ for four different oversaturations $\zeta=0.25,0.5,1.0,2.0$, bottom to top. to do with any line tension effects and that parametrizing them in terms of a generalized Young equation with a line tension term included thus is not justified.

Rather than expressing the result Eq. (11) in terms of the equilibrium contact angle $\theta_{e}$, we can also express it in terms of the equilibrium curvature

$$
R_{e}=\frac{L_{c}}{2 \zeta},
$$

which is constant for one particular experiment (and noninteracting bubbles). Note also that, if shown in terms of $L / L_{c}$, the equilibrium contact angle $\theta_{e}$ or the equilibrium radius of curvature $R_{e} / L_{c}$ are universal for all temperatures, gases, surfaces, liquids, and their corresponding material parameters and only depend on the gas oversaturation $\zeta .^{7}$ Obviously Eqs. (11) and (12) should be tested against experimental results. The main challenge will be to control the gas oversaturation and dedicated setups as the one in Ref. [28] may be required for that.

From Eqs. (11) and (10) we can also see that even for oversaturation $\zeta>0$ an equilibrium does not need to exist: For large $L>L_{c} / \zeta$ the second term in the bracket $[\cdots]$ in Eq. (10) is always dominant and the contact angle of the surface bubble grows without bounds up to bubble detachment- the oversaturation has won against the Laplace pressure. This situation can be compared with the growth of microbubbles out of immersed air-filled hydrophobic micropits (diameter of $10 \mu \mathrm{m}$ ), as studied in Ref. [28] under well controlled oversaturation conditions. It can also be compared to the growth of immersed surface oil droplets in oil oversaturated water, as there the concentration at the oil-water interface is given by the saturation concentration and not by Henry's law (6) and therefore for the surface nanodroplets $L_{c}$ can considered to be zero, i.e., only the second term in the bracket $[\cdots]$ in Eq. (10) exists and for oversaturation $\zeta>0$ one always has droplet growth.

On the other hand, for small enough pinning sites with lateral extension

$$
L<L_{c} / \zeta
$$

stable equilibria and thus stable surface nanobubbles always exist. For very small oversaturation $\zeta$ close to 0 condition (13) does not seem to be a harsh limitation, but in practice obviously the size of the pinning sites the surface offers is limited.

We note that the disjoining pressure [neglected in Eq. (6)] not only slightly modifies the equilibrium contact angle $\theta_{e}$, but also limits the minimal equilibrium contact angle towards some small threshold value $\theta^{*}$, below which the gas-liquid interface is attracted towards the surface so that the three-phase contact line is zipped inwards, leading to an inwards jump of the contact line and an upwards jump of the contact angle. All these effects will quantitatively be worked out and discussed in Ref. [29].

Finally, we actually numerically solve the ODE Eq. (10), see Fig. 4 for one example. For any initial contact angle $\theta_{i}$

\footnotetext{
${ }^{7}$ In practice, strong surface heterogeneities of chemical or geometrical nature may however introduce some nonuniversality and even slightly ovally shaped surface bubbles, i.e., deviations from the spherical cap shape.
} 


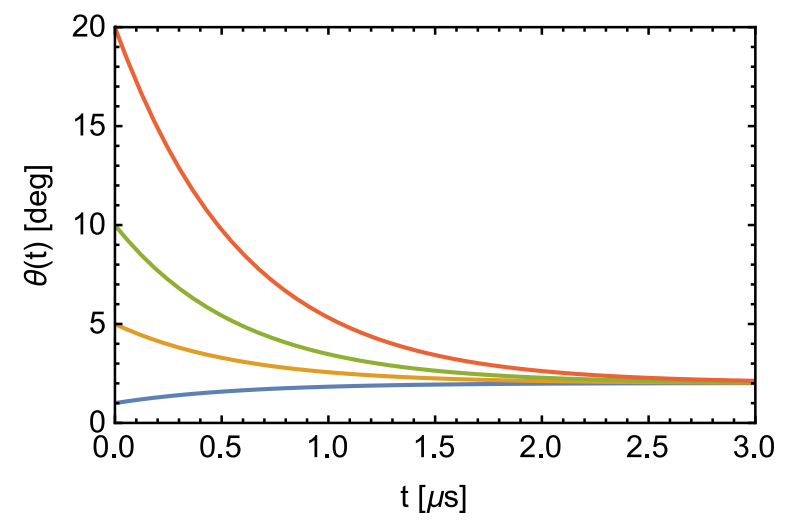

FIG. 4. (Color online) Time evolution of the contact angle for a single surface nanobubble for various initial contact angles $\theta_{0}=1^{\circ}$, $5^{\circ}, 10^{\circ}$, and $20^{\circ}$. The material parameters are chosen for a nitrogen bubble: $D=2 \times 10^{-9} \mathrm{~m}^{2} / \mathrm{s}, c_{s}=0.017 \mathrm{~kg} / \mathrm{m}^{3}, \rho_{g}=1.165 \mathrm{~kg} / \mathrm{m}^{3}$. We picked the lateral extension of the pinning site as $L=100 \mathrm{~nm}$ and a gas oversaturation of $\zeta=1$. Irrespective of the initial contact angle, the surface nanobubble grows towards the equilibrium contact angle $\theta_{e}=\arcsin \left(\zeta L / L_{c}\right)=2.02^{\circ}$.

the stable equilibrium contact angle $\theta_{e}$ is indeed dynamically approached. The time scale associated with this can be read off from Eq. (10) as

$$
\tau=\frac{L^{2}}{4 D} \frac{\rho_{g}}{c_{s}},
$$

which for air bubbles in water is $\tau=86 \mu \mathrm{s}$. As the product of the $\theta$-dependent factors in Eq. (10) happens to be $O\left(10^{2}\right)$, the change in $\theta$ happens even faster, namely on a time scale $O\left(10^{-2} \tau\right)$, as seen in Fig. 4 .

In reality, the shrinkage process does not have to be as ideal as shown in Fig. 4: During shrinkage, the contact line may depin, and either slide or jump to a smaller value of $L$, as suggested in [30] and actually seen for dissolving microdroplets in [31]. As mentioned above, the disjoining pressure plays an important role here [29]. Also during the growth towards $\theta_{e}$ contact line sliding or jumping (now towards a larger $L$ ) may appear. In both cases, the changed lateral extension of the bubble's footprint implies a modified equilibrium contact angle [see Eq. (11)], towards the system will then evolve.

It is illustrative to contrast the stability of the pinned surface nanobubbles to the instability of bulk bubbles already mentioned in the Introduction or the instability of unpinned surface bubbles (i.e., surface bubbles with a constant contact angle $\theta$ ), for which the dynamical equation reads

$$
\dot{L}=\frac{1}{L} \frac{4 D c_{s}}{\rho_{d}} \frac{f(\theta)}{3 g(\theta)}\left(\zeta-\frac{L_{c}}{L} \sin \theta\right) .
$$

For oversaturation $\zeta>0$ also this unpinned case allows for an equilibrium point $(\dot{L}=0)$, namely at

$$
L_{e}=\frac{L_{c}}{\zeta} \sin \theta
$$

but this equilibrium is unstable, as one can easily show with a linear expansion around the equilibrium $L_{e}$, see also the sketch of the phase space for this unpinned situation in Fig. 2(b): Surface nanobubbles with $L<L_{e}$ are pressed out by the Laplace pressure, those with $L>L_{e}$ grow because of the oversaturation, up to detachment from the surface.

We have seen that the crucial parameter for the emergence and stability of pinned surface nanobubbles is the gas oversaturation $\zeta$, which presses gas into the bubble to balance the Laplace pressure. What controls $\zeta$ ? It is the diffusion of gas from the surface to the outer world. The time scale of this process is $\tau_{\text {outer }}=\ell^{2} / D$ (as already elaborated in [8]), which can be rather slow, provided the distance $\ell$ to the outer world is not microscopic. For example, for $\ell=1 \mathrm{~cm}$ one has $\tau_{\text {outer }} \approx 14 \mathrm{~h}$, indeed corresponding to time scales on which changes in the surface nanobubble sizes and partial depinning have been observed [7,32].

To avoid this slow change in the oversaturation $\zeta$ and thus in $\theta_{e}$ the system must be fully decoupled from the outer world, i.e., one must seal the vessel containing the gas oversaturated liquid in contact with the surface. Then the lifetime of the surface nanobubble is infinite. For (large) micrometric surface bubbles (for which, as elaborated above, no stable equilibrium exists as the Laplace pressure is too weak to balance the gas influx) this was achieved in the pressure controlled setup of Ref. [28], which allowed for a precise and long-time control of the saturation level $\zeta$.

Surface nanobubbles not only diffusively communicate with the outer world, but also among each other, as all of them on the one hand affect the gas concentration field $c(\vec{x}, t)$, but on the other hand also react to this field by growth or shrinkage. This may lead to Ostwald ripening, i.e., the growth of large bubbles on the expense of smaller ones, or also the opposite, depending on the relative curvatures of the surface nanobubbles.

In summary, the exact calculation presented in this paper clarifies that the stability of surface nanobubbles and their small contact angle are not "paradoxical." Contact line pinning and gas oversaturation can account for both, reflecting the balance between the gas overpressure due to the oversaturation and the Laplace pressure. The theory quantitatively predicts the equilibrium radius of curvature $R_{e}=L_{c} /(2 \zeta)$ and the equilibrium contact angle $\theta_{e}=\arcsin \left(\zeta L / L_{c}\right)$, which are both universal. In fact, provided one masters precise contact angle measurements, the latter relation may even be used to measure the gas oversaturation $\zeta$. The calculation also reveals what nanobubbles are in contrast to larger bubbles: They have such a small radius of curvature (12) that the Laplace pressure is strong enough to squeeze out and thus compensate for the gas influx due to the gas oversaturation $\zeta>0$ of the liquid. Larger bubbles cannot do this any more and thus grow and finally detach from the wall. Finally, this work offers the perspective to have a fresh view on the problem of contact line pinning and contact angle hysteresis, in particular when using nanostructured surfaces.

We acknowledge useful comments on the manuscript by Sander Huisman, Devaraj van der Meer, Joost Weijs, and Harold Zandvliet. We also acknowledge financial support by an ERC Advanced Grant, by MCEC, sponsored by NWO, and by ARC. 
[1] M. A. Hampton and A. V. Nguyen, Nanobubbles and the nanobubble bridging capillary force, Adv. Colloid Interface Sci. 154, 30 (2010).

[2] V. S. J. Craig, Very small bubbles at surfaces-the nanobubble puzzle, Soft Matter 7, 40 (2011).

[3] J. R. T. Seddon and D. Lohse, Nanobubbles and micropancakes: Gaseous domains on immersed substrates, J. Phys.: Condens. Matter 23, 133001 (2011).

[4] P. Attard, The stability of nanobubbles, Eur. Phys. J. Special Topics (2013), doi:10.1140/epjst/e2013-01817-0.

[5] X. Zhang and D. Lohse, Perspectives on surface nanobubbles, Biomicrofluidics 8, 041301 (2014).

[6] P. S. Epstein and M. S. Plesset, On the stability of gas bubbles in liquid-gas solutions, J. Chem. Phys. 18, 1505 (1950).

[7] X. Zhang, D. Y. C. Chan, D. Wang, and N. Maeda, Stability of interfacial nanobubbles, Langmuir 29, 1017 (2013).

[8] J. H. Weijs and D. Lohse, Why surface nanobubbles live for hours, Phys. Rev. Lett. 110, 054501 (2013).

[9] Y. Liu and X. Zhang, Nanobubble stability induced by contact line pinning, J. Chem. Phys. 138, 014706 (2013).

[10] Y. Liu and X. Zhang, A unified mechanism for the stability of surface nanobubbles: Contact line pinning and supersaturation, J. Chem. Phys. 141, 134702 (2014).

[11] A. Brotchie and X. H. Zhang, Response of interfacial nanobubbles to ultrasound irradiation, Soft Matter 7, 265 (2011).

[12] X. Zhang, H. Lhuissier, C. Sun, and D. Lohse, Surface nanobubbles nucleate microplets, Phys. Rev. Lett. 112, 144503 (2014).

[13] P. G. de Gennes, Wetting: Statics and dynamics, Rev. Mod. Phys. 57, 827 (1985).

[14] L. Leger and J. F. Joanny, Liquid spreading, Rep. Prog. Phys. 55, 431 (1992).

[15] G. D. Nadkarni and S. Garoff, An Investigation of microscopic aspects of contact angle hysteresis: Pinning of the contact line on a single defect, Europhys. Lett. 20, 523 (1992).

[16] D. Bonn, J. Eggers, J. Indekeu, J. Meunier, and E. Rolley, Wetting and spreading, Rev. Mod. Phys. 81, 739 (2009).

[17] J. H. Snoeijer and B. Andreotti, Moving contact lines: Scales, regimes, and dynamical transitions, Annu. Rev. Fluid Mech. 45, 269 (2013).

[18] J. Joanny and P. de Gennes, A model for contact angle hysteresis, J. Chem. Phys. 81, 552 (1984).
[19] P. G. de Gennes, F. Brochard-Wyart, and D. Quere, Capillarity and Wetting Phenomena: Drops, Bubbles, Pearls, Waves (Springer, New York, 2004).

[20] L. Bocquet and E. Charlaix, Nanofluidics, from bulk to interfaces, Chem. Soc. Rev. 39, 1073 (2010).

[21] Y. O. Popov, Evaporative deposition patterns: Spatial dimensions of the deposit, Phys. Rev. E 71, 036313 (2005).

[22] R. D. Deegan, O. Bakajin, T. F. Dupont, G. Huber, S. R. Nagel, and T. A. Witten, Capillary flow as the cause of ring stains from dried liquid drops, Nature (London) 389, 827 (1997).

[23] R. G. Picknett and R. Bexon, The evaporation of sessile or pendant drops in still air, J. Colloid Interface Sci. 61, 336 (1977).

[24] H. Gelderblom, A. G. Marin, H. Nair, A. van Houselt, L. Lefferts, J. H. Snoeijer, and D. Lohse, How water droplets evaporate on a superhydrophobic substrate, Phys. Rev. E 83, 026306 (2011).

[25] A. G. Marin, H. Gelderblom, D. Lohse, and J. H. Snoeijer, Orderto-disorder transition in ring-shaped colloidal stains, Phys. Rev. Lett. 107, 085502 (2011).

[26] N. Kameda and S. Nakabayashi, Size-induced sign inversion of line tension in nanobubbles at a solid/liquid interface, Chem. Phys. Lett. 461, 122 (2008).

[27] C. Xu, S. Peng, G. O. Qiao, V. Gutowski, D. Lohse, and X. Zhang, Nanobubbles on a warmer substrate, Soft Matter 10, 7857 (2014).

[28] O. R. Enriquez, C. Sun, D. Lohse, A. Prosperetti, and D. van der Meer, The quasi-static growth of $\mathrm{CO}_{2}$ bubbles, J. Fluid Mech. 741, R1 (2014).

[29] V. Svetovoy, I. Devic, J. H. Snoeijer, and D. Lohse, The effect of disjoining pressure on surface nanobubbles, Langmuir (to be published).

[30] J. M. Stauber, S. K. Wilson, B. R. Duffy, and K. Sefiane, On the lifetimes of evaporating droplets, J. Fluid Mech. 744, R2 (2014).

[31] X. Zhang, J. Wang, L. Bao, E. Dietrich, R. C. A. van der Veen, S. Peng, J. Friend, H. J. W. Zandvliet, L. Yeo, and D. Lohse, Mixed mode of dissolving immersed microdroplets at a solidwater interface, Soft Matter 11, 1889 (2015).

[32] S. R. German, X. Wu, H. An, V. S. J. Craig, T. L. Mega, and $X$. Zhang, Interfacial nanobubbles are leaky: Permeability of the gas/water interface, ACS Nano 8, 6193 (2014).

[33] R. P. Berkelaar, E. Dietrich, G. A. M. Kip, E. S. Kooij, H. J. W. Zandvliet, and D. Lohse, Exposing nanobubble-like objects to a degassed environment, Soft Matter 10, 4947 (2014). 